\title{
Peristaltic flow with inclined magnetic field and convective boundary conditions
}

\author{
S. Noreen and M. Qasim* \\ Department of Mathematics, COMSATS Institute of Information Technology, Islamabad, Pakistan
}

\begin{abstract}
Peristaltic flow of viscous fluid in an asymmetric inclined channel with heat transfer and inclined magnetic field is examined. The convective boundary conditions have been handled. Complexity of emerging equations is simplified by utilizing long wavelength and low Reynolds number approximation. Variation of emerging parameters embedded in flow system are discussed. It is observed that an increase in Brikman number increases the temperature profile. Further, it is seen that temperature distribution is an increasing function of Biot number at lower wall.
\end{abstract}

Keywords: Convective boundary conditions, inclined magnetic field, heat transfer, peristaltic flow, exact solutions

\section{Introduction}

The study of Peristaltic mechanism has become popular among the researchers during the last four decades because of its vital and extensive applications in physiology and industry. Some of these applications incorporate chyme movement in gastrointestinal tract, swallowing food through esophagus, chyme movement in the gastrointestinal tract, urine transport from kidney to bladder through the ureter, vasomotion of blood vessels in capillaries and arterioles. Since the seminal work of Latham [1] several theoretical and experimental investigations have been carried out in order to understand the peristaltic flows of hydrodynamic fluids under varied assumptions of long wave length, low Reynolds number, small wave amplitude etc. Although the literature on the topic is extensive but few recent investigations can be mentioned by the studies [2-10].

Influence of magnetohydrodynamic (MHD) on peristaltic flow problems have gained significance on the

\footnotetext{
*Corresponding author: M. Qasim, Department of Mathematics, COMSATS Institute of Information Technology, Park Road, Shehzad Town, Islamabad, Pakistan. Tel.: +92 51 9240823; E-mail: mq_qau@yahoo.com.
}

basis of engineering and biomedical applications. Biomagnetic fluid dynamics in medical science has wide range of applications such as stoppage of bleeding during surgeries, cancer tumor treatment, cell separation, targeted transport of drug using magnetic particles as drug carriers etc. Agarwal and Anwaruddin [11] has studied the peristaltic flow of a blood under long wave length and low Reynolds number assumption. Mekheimer [12] reported peristaltic flow of blood under effect of magnetic field in anon-uniform channel. Eldabe et al. [13] analyzed the induced magnetic field effect on peristaltic transport of biviscosity fluid in a non-uniform tube. Mekheimer and Elmaboud [14] had reported the influence of heat and magnetic field on peristaltic transport of Newtonian fluid in a vertical annulus. Vajravelu et al. [15] discussed the combined influence of velocity slip, temperature and concentration jump conditions on MHD peristaltic transport of a Carreau fluid in a non-uniform channel.

The purpose of present research is to discuss the simultaneous effects of an inclined magnetic field and heat transfer with connective boundary conditions [16-20]. To best of our knowledge, this problem has not been investigated yet. The paper is arranged as follows. Sections two and three provides the mathematical 
formulation. Graphical discussion is presented in section four while the concluding remarks are given in section five.

\section{Basic equations}

The Cauchy stress tensor is

$$
\overline{\mathbf{T}}=-p \mathbf{I}+\mu \overline{\mathbf{A}}_{1},
$$

in which $p$ is pressure, $\mathbf{I}$ is identity tensor, $\mu$ is dynamic viscosity and the first Rivilian Ericksin tensor is

$$
\overline{\mathbf{A}}_{1}=\overline{\mathbf{L}}+\overline{\mathbf{L}}^{T}, \quad \overline{\mathbf{L}}=\operatorname{grad} \overline{\mathbf{V}},
$$

where $\overline{\mathbf{V}}$ indicates the velocity.

The following equations can govern the flow under consideration

Continuity equation

$$
\nabla \cdot \mathbf{V}=0
$$

Equation of motion

$\rho \frac{d \mathbf{V}}{d t}=\operatorname{div} \mathbf{T}-\sigma B_{0}^{2} \cos \beta(\bar{U} \cos \beta-\bar{V} \sin \beta)+\rho g \sin \epsilon$

Energy equation

$$
\rho C_{p} \frac{d T}{d t}=\kappa \nabla^{2} T+\text { T.L. }
$$

where $T$ the temperature, $\kappa$ the thermal conductivity, $C_{p}$ the specific heat, $B_{0}$ the applied magnetic field, $g$ is acceleration due to gravity, $\beta$ and $\epsilon$ are inclination angles of magnetic field and channel respectively. The channel walls for heat transfer satisfy convective conditions.

\section{Mathematical formulation}

Peristaltic motion of an incompressible viscous fluid in an inclined asymmetric channel of width $d_{1}+d_{2}$ is investigated. A sinusoidal wave with speed $c$ is propagating on the channel walls. The fluid is electrically conducting and an inclined magnetic field is taken into consideration. The channel walls are non-conducting. We choose rectangular coordinates $(\bar{X}, \bar{Y})$ with $\bar{X}$ in the direction of wave propagation and $\bar{Y}$ transverse to it. The geometry of the wall surfaces are

$$
\begin{array}{ll}
\bar{h}_{1}(\bar{X}, \bar{t}) & \\
=\bar{d}_{1}+\bar{a}_{1} \cos \left(\frac{2 \pi}{\lambda}(\bar{X}-c \bar{t})\right), & \text { upper wall, } \\
\bar{h}_{2}(\bar{X}, \bar{t}) & \\
=-\bar{d}_{2}-\bar{a}_{2} \cos \left(\frac{2 \pi}{\lambda}(\bar{X}-c \bar{t})+\phi\right), & \text { lower wall. }
\end{array}
$$

In above equations $\bar{a}_{1}$ and $\bar{a}_{2}$ are the wave amplitudes. The phase difference $\phi$ varies in the range $0 \leq \phi \leq$ $\pi$. The case $\phi=0$ corresponds to the symmetric channel with waves out of phase and for $\phi=\pi$ the waves are in phase. Further $\bar{a}_{1}, \bar{a}_{2}, \bar{d}_{1}, \bar{d}_{2}$ and $\phi$ satisfy the condition $\bar{a}_{1}^{2}+\bar{a}_{2}^{2}+2 \bar{a}_{1} \bar{a}_{2} \cos \phi \leq\left(\bar{d}_{1}+\bar{d}_{2}\right)^{2}, \lambda$ is the wavelength and $\bar{t}$ is the time. The system is stressed by a constant transverse magnetic field of strength $B_{0}$. The velocity field is written as

$$
\mathbf{V}=[\bar{U}(\bar{X}, \bar{Y}, \bar{t}), \bar{V}(\bar{X}, \bar{Y}, \bar{t}), 0],
$$

where $\bar{U}$ and $\bar{V}$ are the velocity components in the $\bar{X}$ and $\bar{Y}$ directions respectively.

The transformations between the laboratory and wave frames are given by

$$
\begin{aligned}
\bar{x} & =\bar{X}-c \bar{t}, & & \bar{y}=\bar{Y}, \\
\bar{u}(\bar{x}, \bar{y}) & =\bar{U}-c, & & \bar{v}(\bar{x}, \bar{y})=\bar{V},
\end{aligned}
$$

where $(\bar{u}, \bar{v})$ are the velocity components in the wave frame.

With the help of Eqs. (7) and (8), the resulting twodimensional flow equations become

$$
\frac{\partial \bar{u}}{\partial \bar{x}}+\frac{\partial \bar{v}}{\partial \bar{y}}=0
$$

$$
\begin{aligned}
\rho(\bar{u} & \left.\frac{\partial}{\partial \bar{x}}+\bar{v} \frac{\partial}{\partial \bar{y}}\right) \bar{u}+\frac{\partial \bar{p}}{\partial \bar{x}} \\
= & \mu\left(\frac{\partial^{2} \bar{u}}{\partial \bar{x}^{2}}+\frac{\partial^{2} \bar{u}}{\partial \bar{y}^{2}}\right)-\sigma B_{0}^{2} \cos \beta(\bar{U} \cos \beta \\
& \quad-\bar{V} \sin \beta)+\rho g \sin \epsilon, \\
\rho(\bar{u} & \left.\frac{\partial}{\partial \bar{x}}+\bar{v} \frac{\partial}{\partial \bar{y}}\right) \bar{v}+\frac{\partial \bar{p}}{\partial \bar{y}} \\
= & \mu\left(\frac{\partial^{2} \bar{v}}{\partial \bar{x}^{2}}+\frac{\partial^{2} \bar{v}}{\partial \bar{y}^{2}}\right)+\sigma B_{0}^{2} \sin \beta(\bar{U} \cos \beta \\
& -\bar{V} \sin \beta)-\rho g \cos \epsilon,
\end{aligned}
$$




$$
\begin{aligned}
\rho C_{p}\left[\bar{u} \frac{\partial}{\partial \bar{x}}+\bar{v} \frac{\partial}{\partial \bar{y}}\right] \bar{T} \\
=\kappa\left[\frac{\partial^{2} \bar{T}}{\partial \bar{x}^{2}}+\frac{\partial^{2} \bar{T}}{\partial \bar{y}^{2}}\right]+\mu\left[2 \left\{\left(\frac{\partial^{2} \bar{u}}{\partial \bar{x}^{2}}\right)^{2}\right.\right. \\
\left.\left.+\left(\frac{\partial^{2} \bar{v}}{\partial \bar{y}^{2}}\right)^{2}\right\}+\left(\frac{\partial \bar{u}}{\partial y}+\frac{\partial \bar{v}}{\partial \bar{x}}\right)^{2}\right] .
\end{aligned}
$$

The exchange of heat by Newtons law of cooling is given by $[19,20]$

$$
\begin{aligned}
\kappa \frac{\partial \bar{T}}{\partial y}=-\eta_{1}\left(\bar{T}-T_{1}\right) \text { at } & y=h_{1} \\
\kappa \frac{\partial \bar{T}}{\partial y}=-\eta_{0}\left(T_{0}-\bar{T}\right) \text { at } & y=h_{2}
\end{aligned}
$$

where $\eta_{1}, \eta_{0}, T_{1}$ and $T_{0}$ are Biot numbers and temperatures at upper and lower walls respectively. The dimensionless variables and parameters are defined by

$$
\begin{aligned}
& x=\frac{\bar{x}}{\lambda}, y=\frac{\bar{y}}{d_{1}}, t=\frac{c \bar{t}}{\lambda}, p=\frac{d_{1} \bar{p}}{c \lambda \mu}, \\
& d=\frac{d_{2}}{d_{1}}, \gamma=\frac{\bar{T}-T_{0}}{T_{1}-T_{0}}, a=\frac{\bar{a}_{1}}{\bar{d}_{1}}, \\
& \delta=\frac{2 \pi d_{1}}{\lambda}, u=\frac{\bar{u}}{c}, v=\frac{\bar{v}}{c}, \\
& B r=E c \operatorname{Pr}, \operatorname{Pr}=\frac{\mu}{C_{p} \kappa}, M=\sqrt{\frac{\sigma}{\mu}} B_{0} d_{1} \\
& \operatorname{Re}=\frac{c d_{1} \rho}{\mu}, b=\frac{a_{2}}{d_{1}}, F r=\frac{c^{2}}{g d_{1}}, \\
& h_{1}=\frac{\bar{h}_{1}}{d_{1}}, h_{1}=\frac{\bar{h}_{2}}{d_{1}}, Z_{2}=\frac{\eta_{0} d_{1}}{k}, \\
& E c=\frac{c^{2}}{C_{p}\left(T_{\left.1-T_{0}\right)}\right.}, Z_{1}=\frac{\eta_{1} d_{1}}{k}, u=\frac{\partial \Psi}{\partial y}, v=-\delta \frac{\partial \Psi}{\partial x},
\end{aligned}
$$

in which $E c, \operatorname{Pr}, \delta, \operatorname{Re}, M$ and $Z_{1}, Z_{2}$ are the Eckert, Prandtl, wave, Reynolds, Hartman and Biot numbers respectively. Here $p$ is the pressure, $\Psi$ is the stream function. Moreover $T_{0}$ and $T_{1}$ are the temperatures at $y=h_{1}$ and $y=h_{2}$ respectively.

Dimensionless form of Eq. (6) is

$$
\begin{aligned}
& h_{1}=1+a \cos (2 \pi x), \\
& h_{2}=-d-b \cos (2 \pi x+\phi),
\end{aligned}
$$

where $a, b, \phi$ and $d$ satisfy $a^{2}+b^{2}+2 a b \cos \phi \leq$ $(1+d)^{2}$.

Under the application of Eq. (14), equation. (9) is satisfied identically. Eqs. (10) - (13), under assumption of long wavelength and low Reynolds number yield:

$$
\frac{\partial p}{\partial x}=\frac{\partial^{3} \Psi}{\partial y^{3}}-M^{2} \cos \beta\left(\frac{\partial \Psi}{\partial y}+1\right) \cos \beta+\frac{\operatorname{Re}}{F r} \sin \epsilon,
$$

$$
\frac{\partial p}{\partial y}=0
$$

$$
\frac{\partial^{2} \gamma}{\partial y^{2}}+B r\left(\frac{\partial^{2} \Psi}{\partial y^{2}}\right)^{2}=0
$$

where Eq. (18) shows that $p \neq p(y)$ and therefore $p=$ $p(x)$. Application of Eq. (18) on Eq. (17) gives :

$$
\frac{\partial^{4} \Psi}{\partial y^{4}}-M^{2} \cos ^{2} \beta \frac{\partial^{2} \Psi}{\partial y^{2}}=0,
$$

The dimensionless boundary conditions of the problem are

$\Psi=\frac{F}{2}, \frac{\partial \Psi}{\partial y}=-1, \quad \frac{\partial \gamma}{\partial y}+Z_{1} \gamma=0 \quad$ at $\quad y=h_{1}$,

$\Psi=-\frac{F}{2}, \frac{\partial \Psi}{\partial y}=-1, \quad \frac{\partial \gamma}{\partial y}-Z_{2}(\gamma-1)=0$ at $y=h_{2}$,

where $F$ is the dimensionless time mean flow rate in the wave frame. If $\theta$ is the dimensionless time mean flow rate in the laboratory frame then

$$
\theta=F+1+d
$$

with

$$
F=\int_{h_{2}}^{h_{1}} \frac{\partial \Psi}{\partial y} d y
$$

The above set of equations are solved exactly using the the symbolic software Mathematica by DSolve Command. The resulting expressions for stream function $\Psi$, pressure gradient $\frac{d p}{d x}$ and temperature $\gamma$ are given as follows 


$$
\begin{aligned}
& \Psi= \frac{1}{B_{0}}\left[( \operatorname { c o s h } ( \sqrt { B } y ) - \operatorname { s i n h } ( \sqrt { B } y ) ) \left(2\left(F+h_{1}-h_{2}\right)\left(\cosh \left(\sqrt{B}\left(h_{1}+h_{2}\right)\right)+\sinh \left(\sqrt{B}\left(h_{1}+h_{2}\right)\right)\right)\right.\right. \\
& 2\left(F+h_{1}-h_{2}\right)(\cosh (2 \sqrt{B} y)+\sinh (2 \sqrt{B} y))-(2+\sqrt{B} F)\left(h_{1}+h_{2}-2 y\right)\left(\cosh \left(\sqrt{B}\left(y+h_{1}\right)\right)\right. \\
&\left.\left.+\sinh \left(\sqrt{B}\left(y+h_{1}\right)\right)\right)-(-2+\sqrt{B} F)\left(h_{1}+h_{2}-2 y\right)\left(\cosh \left(\sqrt{B}\left(y+h_{2}\right)\right)+\sinh \left(\sqrt{B}\left(y+h_{1}\right)\right)\right)\right] \\
& \frac{d p}{d x}=-2 B B_{0} \cosh \left(\sqrt{B} h_{1}\right)-B^{3 / 2} B_{0} F \cosh \left(\sqrt{B} h_{1}\right)+2 B B_{0} \cosh \left(\sqrt{B} h_{2}\right)-B^{3 / 2} B_{0} F \cosh \left(\sqrt{B} h_{2}\right) \\
& \quad-2 B B_{0} \sinh \left(\sqrt{B} h_{1}\right)-B^{3 / 2} B_{0} F \sinh \left(\sqrt{B} h_{1}\right)+2 B B_{0} \sinh \left(\sqrt{B} h_{2}\right)-B^{3 / 2} B_{0} F \sinh \left(\sqrt{B} h_{2}\right) \\
& \quad-B+\frac{\operatorname{Re} \sin \epsilon}{F r},
\end{aligned}
$$

$$
\begin{aligned}
\gamma= & B_{1}\left[\cosh \left(2 \sqrt{B}\left(h_{1}+h_{2}+y\right)\right)-\sinh \left(2 \sqrt{B}\left(h_{1}+h_{2}+y\right)\right)\left(-4 Z_{1}\left(1+h_{2} Z_{2}-y Z_{2}\right)\right.\right. \\
& \left(\cosh \left(2 \sqrt{B}\left(y+h_{1}+h_{2}\right)\right)+\sinh \left(2 \sqrt{B}\left(y+h_{1}+h_{2}\right)\right)\right)-2 B^{3 / 2} B_{0}^{2} B r\left(F+h_{1}+h_{2}\right)^{2} . \\
& \left(2+h_{1} Z_{1}-y Z_{1}+h_{2} Z_{2}-y Z_{2}\right)\left(\cosh \left(2 \sqrt{B}\left(y+2 h_{1}+h_{2}\right)\right)-\cosh \left(2 \sqrt{B}\left(y+h_{1}+h_{2}\right)\right)\right) \\
& \left.\left.+\sinh \left(2 \sqrt{B}\left(y+2 h_{1}+h_{2}\right)\right)\right)-\sinh \left(2 \sqrt{B}\left(y+2 h_{1}+h_{2}\right)\right)\right)-B B_{0}^{2} B r\left(F+h_{1}-h_{2}\right)^{2} \\
& \left(-Z_{1}+Z_{2}+h_{1} Z_{1} Z_{2}-h_{2} Z_{1} Z_{2}\right)\left(\cosh \left(4 \sqrt{B}\left(h_{1}+h_{2}\right)\right)-\cosh \left(2 \sqrt{B}\left(y+2 h_{1}+h_{2}\right)\right)\right) \\
& \left.\left.\left.-\cosh \left(2 \sqrt{B}\left(y+h_{1}+2 h_{2}\right)\right)\right)+\cosh \left(2 \sqrt{B}\left(2 y+h_{1}+h_{2}\right)\right)\right)+\sinh \left(4 \sqrt{B}\left(h_{1}+h_{2}\right)\right)\right) \\
& \left.-\sinh \left(2 \sqrt{B}\left(2 h_{1}+h_{2}+y\right)\right)\right),
\end{aligned}
$$

$$
\begin{aligned}
B_{0}= & 2\left(\left(-2+\sqrt{B} h_{1}-\sqrt{B} h_{2}\right)\left(\cosh \left(2 \sqrt{B} h_{1}\right)+\sinh \left(2 \sqrt{B} h_{1}\right)\right)+\left(2+\sqrt{B} h_{1}-\sqrt{B} h_{2}\right)\right. \\
& \left(\cosh \left(2 \sqrt{B} h_{2}\right)+\sinh \left(2 \sqrt{B} h_{2}\right)\right),
\end{aligned}
$$

$$
B_{1}=\frac{1}{4\left(-Z_{1}+Z_{2}+h_{1} Z_{1} Z_{2}-h_{2} Z_{1} Z_{2}\right)}, B=M^{2} \cos ^{2} \beta
$$

The dimensionless expressions of pressure rise $\Delta P_{\lambda}$ is

$$
\Delta P_{\lambda}=\int_{0}^{1} \frac{d p}{d x} d x .
$$

\section{Discussion}

Our interest in this section is to examine the variations of Brinkman number $\mathrm{Br}$, channel inclination $\epsilon$, Hartman number $M$, phase difference $\phi$, inclines magnetic field parameter $\beta$ and wave amplitude $a$ on the pressure gradient $d p / d x$, pressure rise $\Delta P_{\lambda}$, temperature distribution $\gamma$ and velocity $u$. For this purpose the Figs. 1(a-c)-4(a-f) have been plotted.

The behaviors of $\beta, \epsilon$ and $a$ on the axial pressure gradient is shown in Fig. 1(a-c). It is evident that magnitude of $d p / d x$ has an opposite effects for $\beta$ and $\epsilon$.
It decreases when $\beta$ increases. It is also clear that $d p / d x$ is directly proportional to $a$. Further, Fig. 1(a-c) depict that in the wider part of channel the pressure gradient is small and flow can easily pass without the application of large pressure gradient. However in the narrow part of the channel a much larger pressure gradient is required to maintain the same flux to pass it.

The variation of pressure rise $\Delta P_{\lambda}$ against mean flow rate $\theta$ for different values of $\beta, a$ and $M$ is plotted in Fig. 2(a-c). Pumping action is due to the dynamic pressure exerted by the walls on the fluid trapped between the contraction region. Inclined magnetic field parameter $\beta$ has opposite behavior on pressure rise when compared with the Hartman number $M$. Figure $2 \mathrm{~b}$ shows that influence of $a$ on $\Delta p_{\lambda}$ is similar to that of $M$ It is noticed that an increase in $M$ causes an increase in the pressure rise (Fig. 2c). In the pumping region 

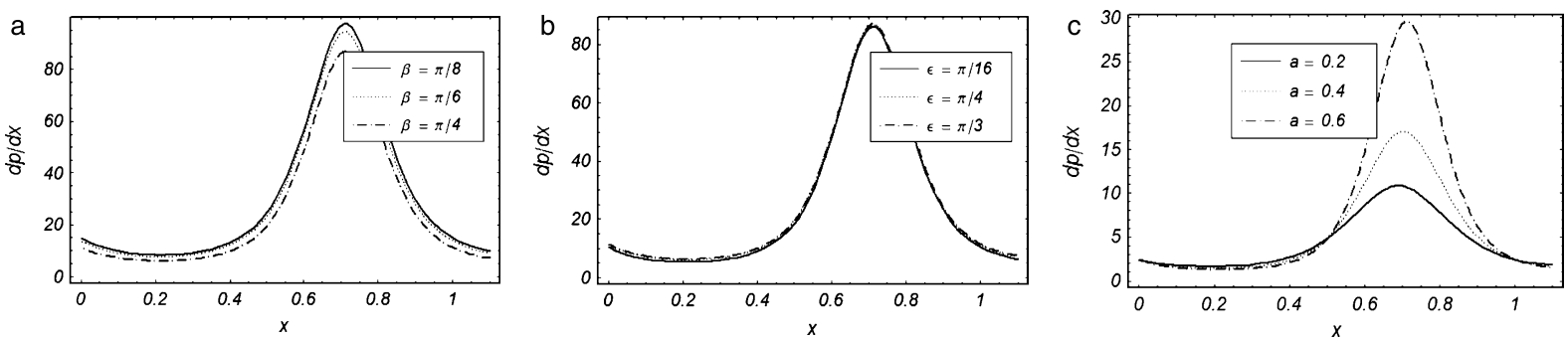

Fig. 1. a) The pressure gradient $\mathrm{dp} / \mathrm{dx}$ versus $\mathrm{x}$ for $\phi=\pi / 6, \theta=5.5, \mathrm{a}=0.6, \mathrm{~b}=0.5, \mathrm{M}=2, \varepsilon=\pi / 4, \operatorname{Re}=2$, $\mathrm{Fr}=1.3$ and $\mathrm{d}=1.1$, b) The pressure gradient dp/dx versus $\mathrm{x}$ for $\phi=\pi / 6, \theta=5.5, \mathrm{a}=0.6, \mathrm{~b}=0.5, \mathrm{M}=2, \beta=\pi / 8, \mathrm{M}=2 \mathrm{Re}=2, \mathrm{Fr}=1.3$ and $\mathrm{d}=1.1, \mathrm{c}) \mathrm{The}$ pressure gradient $\mathrm{dp} / \mathrm{dx}$ versus $\mathrm{x}$ for $\phi=\pi / 6, \theta=5.5, \varepsilon=\pi / 4, \mathrm{~b}=0.5, \mathrm{a}=0.6, \beta=\pi / 4, \operatorname{Re}=2$, $\mathrm{Fr}=1.3$ and $\mathrm{d}=1.1$.
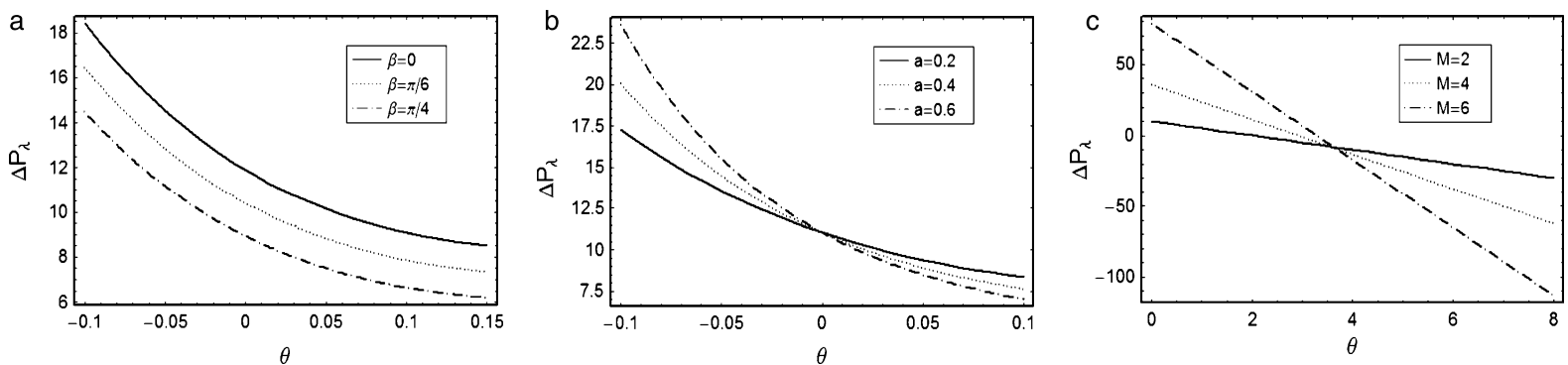

Fig. 2. a) The pressure rise $\Delta P_{\lambda}$ versus $\theta$ for $\phi=\pi / 6, \theta=-5.5, \mathrm{a}=0.6, \mathrm{~b}=0.5, \mathrm{M}=2, \varepsilon=\pi / 4, \mathrm{Re}=2, \mathrm{Fr}=1.3$ and $\left.\mathrm{d}=1.1, \mathrm{~b}\right) \mathrm{The}$ pressure rise $\Delta P_{\lambda}$ versus $\theta$ for $\phi=\pi / 6, \theta=5.5, \varepsilon=\pi / 4, \mathrm{~b}=0.5, \mathrm{M}=2, \beta=\pi / 4, \mathrm{M}=2 \mathrm{Re}=2, \mathrm{Fr}=1.3$ and $\left.\mathrm{d}=1.1, \mathrm{c}\right)$ The pressure rise $\Delta P_{\lambda}$ versus $\theta$ for $\phi=\pi / 6, \theta=-5.5, \varepsilon=\pi / 4, \mathrm{~b}=0.5, \mathrm{a}=0.6, \beta=\pi / 4, \operatorname{Re}=2, \mathrm{Fr}=1.3$ and $\mathrm{d}=1.1$.
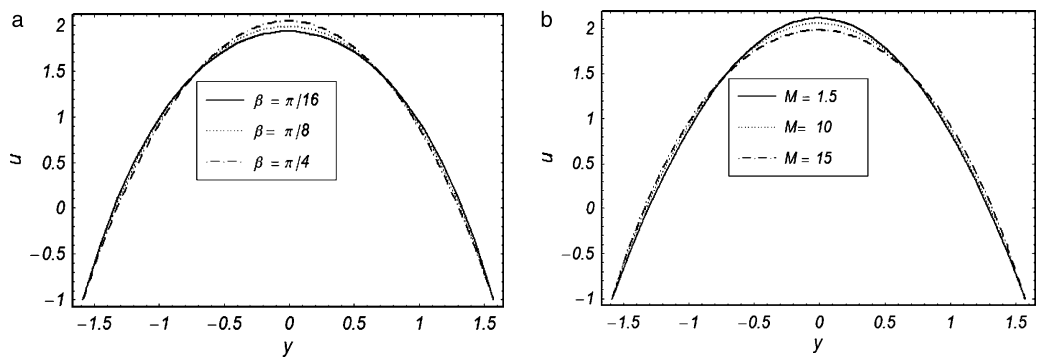

Fig. 3. a) The velocity function $u$ versus $y \mathrm{a}=0.6, \theta=5.5, \phi=\pi / 6, b=0.5, x=0.2, M=1.5$ and $d=1.1$, b) The velocity function $u$ versus $y$ for $\mathrm{a}=0.6, \theta=5.5, \phi=\pi / 6, b=0.5, x=0.2, M=1.5$ and $d=1.1$.

$\left(\Delta p_{\lambda}>0\right)$ the pumping rate increases by increasing $M$. The free pumping rate $\left(\Delta p_{\lambda}=0\right)$ increases by increasing $M$. In copumping $\left(\Delta p_{\lambda}<0\right)$ the pumping rate decreases by increasing $M$.

Figure $3(a, b)$ has been plotted in order to study the effects of $\beta$ and $M$ on the velocity function $u$. Figs. $3 \mathrm{a}$ depicts that the magnitude of velocity function $u$ increases by increasing $\beta$ parameter at the centre of channel. Figure $3 \mathrm{~b}$ depicts a behavior opposite to $\beta$.

The variation of temperature distribution $\gamma$ against $y$ is drawn in Fig. 4(a-f). The following observations are worth noted.
Figure $4(\mathrm{a}-\mathrm{f})$ is plotted to observe the influence of $\beta, B r, M, \phi, Z_{1}$ and $Z_{2}$ on the temperature distribution. Temperature distribution decreases by increasing $M$ and $Z_{1}$. The temperature distribution increases by increasing values of Brinkman number $\mathrm{Br}$, inclined magnetic field $\beta$ and phase difference $\phi$ and Biot number $Z_{2}$.

\section{Conclusion}

A theoretical study is made to observe the MHD flow characteristics of viscous fluid under the influence 

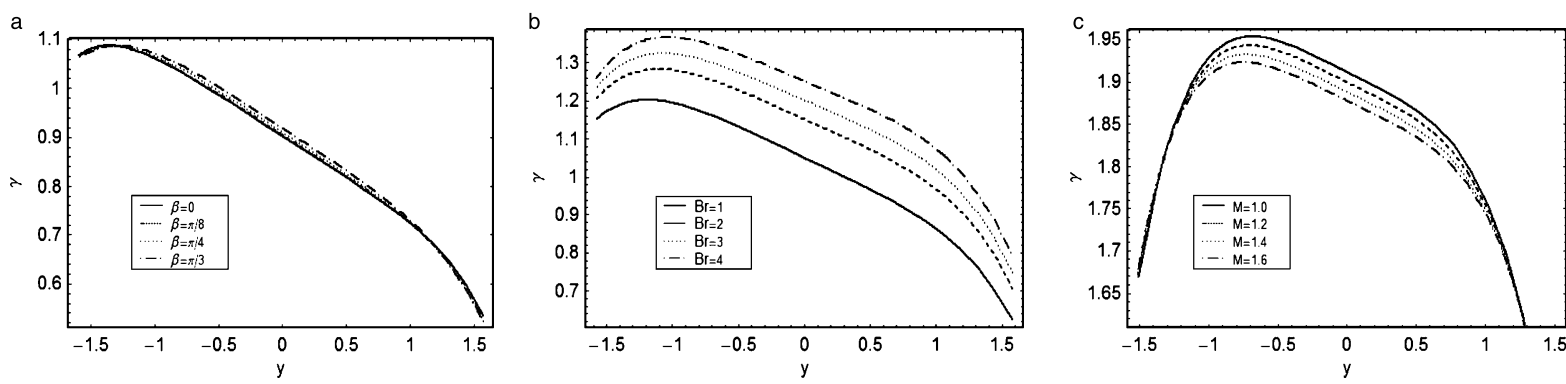

e

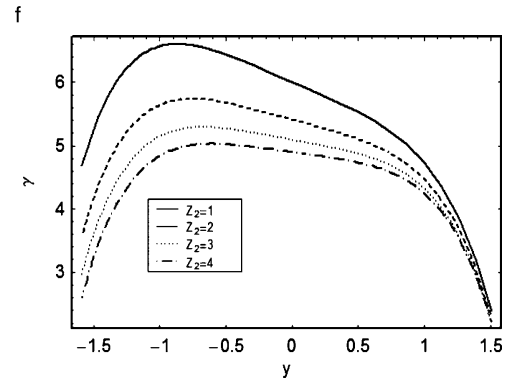

Fig. 4, a) The temperature distribution $\gamma$ versus $y$ for $\mathrm{a}=0.6, \theta=0.3, \phi=\pi / 8, \mathrm{~b}=0.5, \mathrm{x}=0.2, M=1.5, \mathrm{~d}=1.1, \mathrm{Br}=1, \mathrm{Z}_{1}=1$ and $\mathrm{Z}$ $=3, \mathrm{~b})$ The temperature distribution $\gamma$ versus $y \beta=0.05, \mathrm{a}=0.6, \theta=0.3, \phi=\pi / 8, \mathrm{~b}=0.5, \mathrm{x}=0.2, \mathrm{M}=1.5, \mathrm{~d}=1.1, \mathrm{Z}_{1}=1$ and $\mathrm{Z}_{2}$ $=3, \mathrm{c})$ The temperature distribution $\gamma$ versus $y \beta=0.05, \mathrm{a}=0.6, \theta=0.3, \phi=\pi / 8, \mathrm{~b}=0.5, \mathrm{x}=0.2, \mathrm{Br}=1, \mathrm{~d}=1.1, \mathrm{Z}_{1}=1$ and $\mathrm{Z}_{2}=3$, d) The temperature distribution $\gamma$ versus $y \beta=0.05, \mathrm{a}=0.6, \theta=0.3, \mathrm{M}=1.5, \mathrm{~b}=0.5, \mathrm{x}=0.2, \mathrm{Br}=1, \mathrm{~d}=1.1, \mathrm{Z}_{1}=1$ and $\mathrm{Z}_{2}=3$. e) $\mathrm{The}$ temperature distribution $\gamma$ versus y $\beta=0.05, \mathrm{a}=0.6, \theta=0.3, \mathrm{M}=1.5, \mathrm{~b}=0.5, \mathrm{x}=0.2, \mathrm{Br}=1, \mathrm{~d}=1.1, \phi=\pi / 8$ and $\left.\mathrm{Z}_{2}=3, \mathrm{f}\right) \mathrm{The}$ temperature distribution $\gamma$ versus y $\beta=0.05, \mathrm{a}=0.6, \theta=0.3, \mathrm{M}=1.5, \mathrm{~b}=0.5, \mathrm{x}=0.2, \mathrm{Br}=1, \mathrm{~d}=1.1, \phi=\pi / 8$ and $\mathrm{Z}_{1}=1$.

of inclined magnetic field and convective boundary conditions. The effects of different parameters on the velocity and temperature profiles are shown. An exact solution is constructed. The following observations are made.

- An increase in the values of $\beta$ and $M$ have opposite effects on the velocity $u$ in a qualitative sense.

- The temperature profile increases by increasing $B r, \beta, \phi$ and $Z_{2}$ and while it decreases when $M, Z_{1}$ are increased.

- An increase in Brikman number $\mathrm{Br}$ increases the temperature profile.

- Higher values of $d p / d x$ corresponds to smaller values of inclined magnetic field $\beta$.

\section{References}

[1] T.W. Latham, Fluid motion in a peristaltic pump, MIT Cambridge MA, 1966.

[2] Kh., S. Mekheimer and Y. Abd elmaboud, Peristaltic flow of a couple stress fluid in an annulus: Application of an endoscope, Physica A 387 (2008), 2403-2415.

[3] S. Nadeem and S. Akram, Influence of inclined magnetic field on peristaltic flow of a Williamson fluid model in an inclined symmetric or asymmetric channel, Mathematical and Computer Modelling 52 (2010), 107-119.

[4] D. Tripathi, Study of transient peristaltic heat flow through a finite porous channel, Mathematical \& Computer Modelling 57 (2013), 1270-1283.

[5] D. Tripathi and O.A. Beg, A study on peristaltic flow of nanofluids: Application in drug delivery systems, Int J Heat \& Mass Transfer 70 (2014), 61-70.

[6] M. Saleem and A. Haider, Heat and mass transfer on the peristaltic transport of non-Newtonian fluid with creeping flow, Int J Heat \& Mass Transfer 68 (2014), 514-526.

[7] S. Noreen, T. Hayat, A. Alsaedi and M. Qasim, Mixed convection heat and mass transfer in peristaltic flow, Indian Journal of Physics 87(9) (2013), 889-896.

[8] Y. Abd elmaboud and Kh. S. Mekheimer, Non-linear peristaltic transport of a second-order fluid through a porous medium, Applied Mathematical Modelling 35 (2011), 2695-2710.

[9] Y. Abd elmaboud, KH.S. Mekheimer and A. Abdellartif, Thermal properties of couple-stress fluid flow in an asymmetric channel with peristalsis, J Heat Transfer 135(4) 044502 (2013).

[10] S. Noreen and M. Qasim, Peristaltic flow of MHD EryingPowell fluid, The European Physical Journal Plus 128(8) (2013), 1-10.

[11] H.L. Agarwal and B. Anwaruddin, Peristaltic flow of blood in a branch, Ranchi Univ Math J 15 (1984), 111-118.

[12] Kh. S. Mekheimer, Peristaltic flow of blood under the effect of magnetic field in a non-uniform channels, Appl Math Comput 153 (2004), 763-777. 
[13] N.T.M. Eldabe, M.F. El Sayed, A.Y. Ghaly and H.M. Sayed, Peristaltically induced transport of MHD biviscosity fluid in a non-uniform tube, Physica A 383 (2007), 253-266.

[14] Kh. S. Mekheimer and Y.A. Elmaboud, The influence and heat transfer and magnetic field on peristaltic transport of a magnetic field on peristaltic transport of Newtonian fluid in a vertical annulus: Application of an endoscope, Phys Lett A 372 (2008), 1657-1665.

[15] K. Vajravelu, S. Sreenadh and R. Saravana, Combined influence of velocity slip, temperature and concentration jump conditions on MHD peristaltic transport of a Carreau fluid in a non-uniform channel, Applied Mathematics and Computation 225 (2013), 656-676.

[16] S. Srinivas, T. Malathy and A. Subramanyam Reddy, A note on thermal-diffusion and chemical reaction effects on MHD pulsating flow in a porous channel with slip and convective boundary conditions, J King Saud Univ - Eng Sci, In Press.
[17] O.D. Makinde, T. Chinyoka and L. Rundora, Unsteady flow of a reactive variable viscosity non-Newtonian fluid through a porous saturated medium with asymmetric convective boundary conditions, Computers \& Mathematics with Applications 62 (2011), 3343-3352.

[18] L. Rundora and O.D. Makinde, Effects of suction/injection on unsteady reactive variable viscosity non-Newtonian fluid flow in a channel filled with porous mediumand convective boundary conditions, Journal of Petroleum Science and Engineering 108 (2013), 328-335.

[19] F.M. Abbasi, T. Hayat and B. Ahmad, Peristaltic flow in an asymmetric channel with convective boundary conditions and Joule heating, J Cent South Univ 21 (2014), 1411-1416.

[20] T. Hayat, H. Yasmeen, B. Ahmed and B. Chen, Simultaneous effects of convective conditions and nanoparticles on peristaltic motion, J Molecular Liquids 193 (2014), 74-82. 

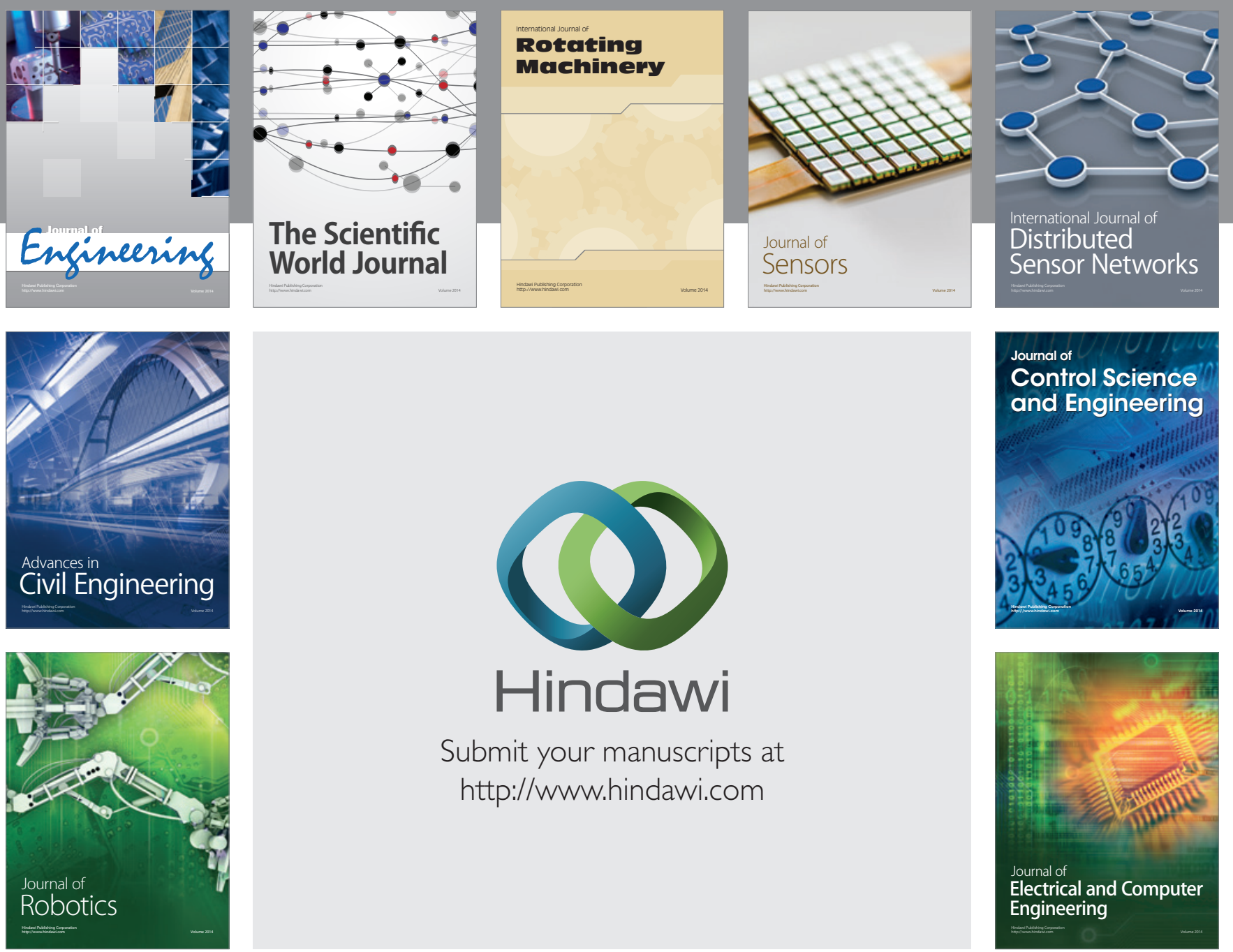

Submit your manuscripts at

http://www.hindawi.com
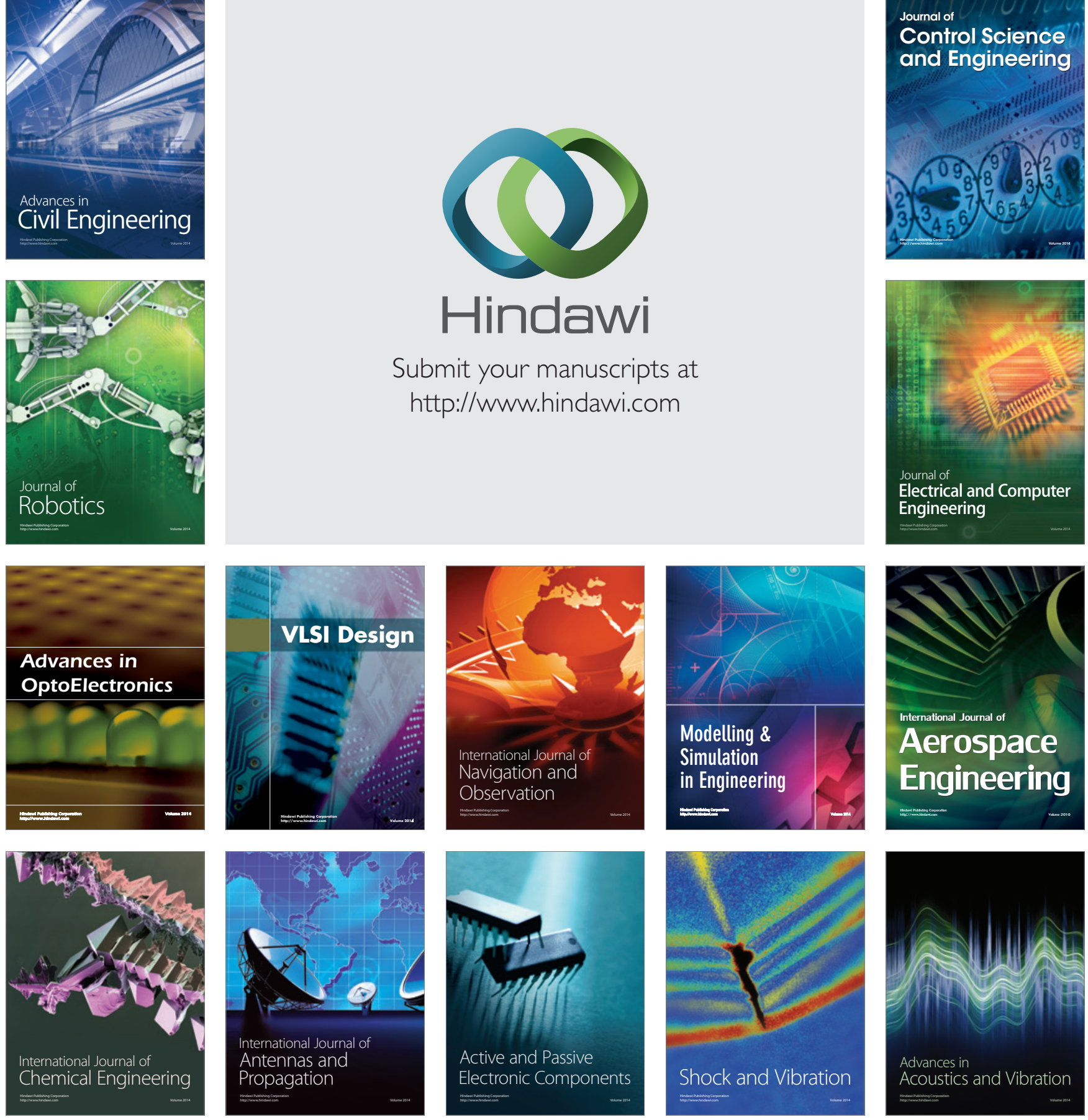